\title{
Structural brain correlates of defective gesture performance in schizophrenia
}

Katharina Stegmayer $^{\mathrm{a}}$, Stephan Bohlhalter ${ }^{\mathrm{b}, \mathrm{c}}$, Tim Vanbellingen ${ }^{\mathrm{b}, \mathrm{c}}$, Andrea Federspiel ${ }^{\mathrm{a}}$, Jeanne Moor $^{\mathrm{a}}$, Roland Wiest ${ }^{\mathrm{d}}$, René Müri ${ }^{\mathrm{c}, \mathrm{e}}$, Werner Strik ${ }^{\mathrm{a}}$, Sebastian Walther ${ }^{\mathrm{a},{ }^{*}}$

${ }^{a}$ University Hospital of Psychiatry, Bern, Switzerland

${ }^{\mathrm{b}}$ Neurology and Neurorehabilitation Center, Kantonsspital Luzern, Switzerland

${ }^{\mathrm{c}}$ Department of Clinical Research, University Hospital, Inselspital, Bern, Switzerland ${ }^{\mathrm{d}}$ Institute of Neuroradiology, Inselspital, Bern, Switzerland

${ }^{\mathrm{e}}$ Department of Neurology, University Hospital, Inselspital, Bern, Switzerland

*Corresponding author: Dr. Sebastian Walther, M.D., University Hospital of Psychiatry, Bolligenstrasse 111, 3000 Bern 60, Switzerland, E-mail: walther@puk.unibe.ch 


\section{Abstract}

Introduction: The neural correlates of impaired performance of gestures are currently unclear. Lesion studies showed variable involvement of the ventro-dorsal stream particularly left inferior frontal gyrus (IFG) in gesture performance on command. However, findings cannot be easily generalized as lesions may be biased by the architecture of vascular supply and involve brain areas beyond the critical region. The neuropsychiatric syndrome of schizophrenia shares apraxic-like errors and altered brain structure without macroanatomic lesions. Schizophrenia may therefore qualify as a model disorder to test neural correlates of gesture impairments.

Methods: We included 45 schizophrenia patients and 44 healthy controls in the study to investigate the structural brain correlates of defective gesturing in schizophrenia using voxel based morphometry. Gestures were tested in two domains: meaningful gestures (transitive and intransitive) on verbal command and imitation of meaningless gestures. Cut-off scores were used to separate patients with deficits, patients without deficits and controls. Group differences in GM volume were explored in an ANCOVA.

Results: Patients performed poorer than controls in each gesture category $(p<0.001)$. Patients with deficits in producing meaningful gestures on command had reduced gray matter (GM) predominantly in left IFG, with additional involvement of right insula and anterior cingulate cortex. Patients with deficits differed from patients without deficits in right insula, inferior parietal lobe and superior temporal gyrus.

Conclusions: Impaired performance of meaningful gestures on command was linked to volume loss predominantly in the praxis network in schizophrenia. Thus, the behavioral similarities between apraxia and schizophrenia are paralleled by structural alterations. However, few associations between behavioral impairment and structural brain alterations appear specific to schizophrenia.

\section{keywords}

praxis network; intransitive; transitive; meaningless; pantomime; action planning 


\section{Introduction}

Hand gestures are important for nonverbal communication (Cartmill, Beilock, \& GoldinMeadow, 2012). In addition, gestures allow studying cerebral action representation in health and disease, such as apraxia (Steele, Ferrari, \& Fogassi, 2012). Gesturing can be elicited either by a verbal command that specifies the content of the gesture or by demonstration and subsequent imitation of the gesture. The gesture may be meaningful or meaningless. Meaningful gestures may either have a conventionally agreed meaning (e. g., thumbs up for "okay") or refer to tools and their actions (e. g., the typical movement of hammering). Out of the possible combinations of mode of elicitation (verbal command versus imitation) and content (meaningless, tool use, conventional meaning) we concentrate on imitation of meaningless gestures on the one hand and production of meaningful gestures on verbal command on the other. To facilitate the comparison with other studies using different nomenclature we note that gestures referring to tool use have also been classified "pantomime of tool use" and "transitive gestures", whereas gestures with a conventional meaning have been termed "emblems" and "intransitive" gestures.

Action planning and its neuroanatomical representation are lively debated in behavioral and neuroimaging studies. Two action systems are currently discussed, including a ventro-dorsal stream running from primary visual cortex to middle temporal gyrus to the inferior parietal lobe (IPL) to the ventral premotor cortex (PMv) - and a dorso-dorsal stream connecting primary visual cortex, V6, intraparietal sulcus (IPS), superior parietal lobe (SPL) and dorsal premotor cortex (PMd) (Binkofski \& Buxbaum, 2013; Buxbaum \& Kalenine, 2010; Rizzolatti $\&$ Matelli, 2003). The ventro-dorsal stream is thought to store long-term action knowledge critical for object use and pantomime of object use, while the dorso-dorsal stream is used for immediate online control of motor acts (Binkofski \& Buxbaum, 2013). However, studies on cerebral action representation are not entirely conclusive across patient groups and controls. Functional MRI (fMRI) studies in healthy subjects support the general functional distinction in ventro-dorsal stream for meaningful gestures on verbal command and dorso-dorsal stream for imitation, albeit the distinction is not strict. Particularly, fMRI studies demonstrated a bilateral but left lateralized fronto-temporo-parietal network engaged in planning of meaningful gestures on command (Bohlhalter et al., 2009; Fridman et al., 2006; JohnsonFrey, Newman-Norlund, \& Grafton, 2005; Kroliczak \& Frey, 2009; Vry et al., 2015). Performance of both intransitive and transitive gestures on verbal command has elicited brain activation in overlapping brain areas with some studies reporting less lateralization in transitive gestures (Bohlhalter et al., 2009; Fridman et al., 2006). Furthermore, the contrast 
transitive vs. intransitive gestures demonstrated activation within the left inferior part of the inferior frontal gyrus (IFG) (Fridman et al., 2006), the left PMd and inferior parietal cortex (Kroliczak \& Frey, 2009). Results of a combined fMRI and fiber tracking study suggest that performance of transitive gestures on command engages the ventro-dorsal route and additionally the ventral route via IFG (Vry et al., 2015). In healthy controls the imitation of hand gestures is associated with neural activity in bilateral IFG, PMd, IPL and SPL (Horan et al., 2014; Menz, McNamara, Klemen, \& Binkofski, 2009; Muhlau et al., 2005; Vry et al., 2015). Even though these fMRI studies have been informative on neural action representation under normal conditions, they do not allow inferences on the nature of the disturbances seen in abnormal gesturing, such as apraxia.

Lesion mapping studies of predominantly left hemisphere stroke patients revealed that poor imitation performance was associated with lesions in parietal and posterior temporal cortices (Buxbaum, Shapiro, \& Coslett, 2014; Hoeren et al., 2014b; Mengotti et al., 2013), less often with lesions in the IFG or insula (Weiss et al., 2014), which have been suggested to incorporate specificity of body parts (Goldenberg \& Karnath, 2006). Most studies tested imitation of meaningless gestures, but some also investigated imitation of tool related (transitive) gestures. Buxbaum and colleagues proposed that the kinematics of imitative gestures relied on IPL and SPL, the tool related knowledge in transitive gestures instead relied on posterior temporal cortex integrity (Buxbaum et al., 2014).

Lesion mapping studies also focused on transitive gestures on verbal command. Impaired performance of such tool-related gestures was linked to lesions in left IFG, insula, premotor cortex, hippocampus, IPL and lateral occipitotemporal cortex (Goldenberg, Hermsdorfer, Glindemann, Rorden, \& Karnath, 2007; Hoeren et al., 2014b; Manuel et al., 2013; Weiss et al., 2014). However, some reports suggested specific contributions, e.g. content errors and IFG lesions, spatial errors and IPL lesions (Manuel et al., 2013) or posture in tool use and lesions in middle temporal gyrus (MTG) and inferior temporal gyrus (ITG) (Buxbaum et al., 2014). Lesion mapping studies have not attempted to investigate performance of meaningless or intransitive gestures on verbal command.

Brain lesions due to ischemic stroke cover a wide range of affected brain tissue, including gray and white matter. Lesion mapping studies are therefore limited in their specificity. Even though the gesture deficit is clear, misattributions of the causes are possible, as the brain lesions include more areas than those critical for correct gesturing. Furthermore, in contrast to most fMRI investigations in healthy subjects, lesion studies suggest that gesture performance was exclusively linked to left hemispheric networks. 
While fMRI studies in healthy subjects have informed on particular brain regions they failed to mimic defective gesturing. In contrast, lesion mapping studies provide precise information on the gesture deficit but lesion mapping is less specific and contaminated by the anatomical boundaries of ischemic stroke. The neural correlates of defective gesturing may be investigated in a model disorder combining subtle structural brain and gestural deficits.

Schizophrenia is characterized by poor social interaction, which contributes to the devastating functional outcome (Schmidt, Mueller, \& Roder, 2011). Particularly, nonverbal communication is disturbed, both during perception and expression of socially relevant information (Green et al., 2012; Lavelle, Healey, \& McCabe, 2013; Pinkham, 2014). Gesture is particularly interesting to understand social cognitive deficits in schizophrenia (Walther \& Mittal, 2016). Patients are impaired in action imitation, particularly imitation of finger and hand gestures (Matthews, Gold, Sekuler, \& Park, 2013; Park, Matthews, \& Gibson, 2008). These deficits have been linked to aberrant function of the mirror neuron system (Thakkar, Peterman, \& Park, 2014). The accuracy of hand gestures is severely impaired in schizophrenia, particularly when performed on verbal command, but less impaired during imitation (Walther et al., 2015; Walther, Vanbellingen, Muri, Strik, \& Bohlhalter, 2013a, $2013 \mathrm{~b}$ ). Indeed, 40-60\% of patients are impaired in gesture performance on verbal command, while 23-33\% present defective imitation of hand gestures (Walther et al., 2013a, 2013b). The most frequent errors occur when performing meaningless gestures on verbal command (Walther et al., 2013a). Poor performance was linked to frontal lobe dysfunction, impaired working memory and motor abnormalities (Walther et al., 2015; Walther et al., 2013b). Gray matter alterations have been demonstrated in schizophrenia patients in meta-analyses also in praxis relevant brain areas, including the bilateral IFG and insula, superior temporal gyrus (STG) and parietal lobe (Bora et al., 2011; Haijma et al., 2013). Furthermore, aberrant white matter ultrastructure was reported in long association fibers such as the superior longitudinal fasciculus and uncinate fasciculus as well as in interhemispheric and projection fibers of the frontal lobe (Bora et al., 2011; Peters, Blaas, \& de Haan, 2010; Walther et al., 2011a).

Schizophrenia may present both, a behavioral gesture deficit as well as brain alterations in gray and white matter within the praxis relevant network. Thus, the disorder may serve as a neuropsychiatric model of defective pantomime without macroanatomic brain lesions. As imitation of gestures is less affected in schizophrenia, the model will be confined to deficits in pantomime. Patients with apraxia usually suffer from impairments in tool use, gesturing, fine 
and gross motor function due to structural brain lesions. Hand gesture performance is impaired during both imitation and pantomime; errors include spatial and temporal errors as well as content errors (e.g. body part as object) (Vanbellingen et al., 2010). In apraxia impairments in action planning are multimodal as indicated by altered visual scanning and perception of action-related sounds (Pazzaglia, Pizzamiglio, Pes, \& Aglioti, 2008; Vanbellingen et al., 2015). Schizophrenia patients share the same type of gesture errors, but the extent of the impairment is less pronounced than in apraxia (Vanbellingen et al., 2010; Walther et al., 2013a). In addition, schizophrenia is associated with subtle fine and gross motor impairments, frontal lobe dysfunction and impaired social cognition (Walther et al., 2015; Walther et al., 2013a).

To investigate the structural brain correlates of defective gesturing in schizophrenia, we focused on group differences in gray matter volume according to gesture performance, i.e. the difference between patients with gesture deficits vs. patients without gesture deficits vs. healthy controls. We expected reduced gray matter (GM) volume within the ventro-dorsal stream (particularly in the left IFG) in schizophrenia patients with apparent impairments of gesture performance on verbal command compared to patients with intact gesture performance and healthy subjects. Likewise, we hypothesized reduced GM volume within the parietal cortex in schizophrenia patients with defective gesture imitation. Finally, we explored the association of impaired gesture performance on verbal command and GM volume in patients for each category of meaningful gestures, i.e. intransitive (conventional meaning) and transitive (tool use). We hypothesized that impaired performance of intransitive gestures would be related to GM volume reduction in brain areas processing language/semantic content such as MTG and STG. Furthermore, impaired performance of transitive gestures on verbal command would be related to reduced GM volume in IFG and SPL. 


\section{Materials and methods}

\subsection{Participants}

In total, 45 patients with schizophrenia spectrum disorders according to DSM-5 criteria ( schizophrenia $n=34$, schizoaffective disorder $n=2$, schizophreniform disorder $n=9$ ) were recruited at the inpatient and outpatient departments of the University Hospital of Psychiatry, Bern. In addition, 44 healthy control subjects were recruited via advertisement and among staff and students. Controls were matched for age, gender, and duration of education. All participants were right-handed according to the Edinburgh handedness inventory (Oldfield, 1971). General exclusion criteria were substance abuse or dependence other than nicotine; history of motor impairments such as dystonia, idiopathic parkinsonism, multiple sclerosis or stroke; history of head trauma with concurrent loss of consciousness; history of electroconvulsive treatment. Exclusion criteria for controls comprised history of any psychiatric disorder, as well as any first-degree relatives with schizophrenia or schizoaffective disorder. All participants were interviewed with the Mini International Neuropsychiatric Interview (MINI) (Sheehan et al., 1998). Patients were further interviewed with the Comprehensive Assessment of Symptoms and History (CASH) (Andreasen, Flaum, \& Arndt, 1992).

All but four patients were on antipsychotic medication, mean chlorpromazine equivalents (CPZ) were $401.29 \pm 351.56 \mathrm{mg}$. Psychopathology was assessed with the Positive And Negative Syndrome Scale (PANSS) (Kay, Fiszbein, \& Opler, 1987). Patients' mean scores were $18.36 \pm 6.48$ for the positive subscale, $18.42 \pm 5.13$ for the negative subscale, and 72.93 \pm 17.38 for the PANSS total score. Furthermore, patients had parkinsonian symptoms as determined by the Unified Parkinson's Disease Rating scale (Fahn, Elton, \& Members, 1987) motor part $(7.35 \pm 7.42)$. In addition, some of the patients suffered from dyskinesia as assessed by the Abnormal Involuntary Movement Scale (AIMS) (Guy, 1976) with average scores of $2.33 \pm 3.90$.

The protocol was approved by the local ethics committee. All participants provided written informed consent.

\subsection{Gesture tests}

We applied the Test of Upper Limb Apraxia (TULIA) (Vanbellingen et al., 2010) in the dominant right arm. The TULIA is a comprehensive assessment tool of hand and finger gestures in two domains termed imitation (performance following demonstration by the examiner) and pantomime (performance following verbal command). The TULIA includes 
three semantic categories, i.e. meaningless novel gestures, intransitive (conventional meaning) gestures and transitive (tool-related) gestures. Performance was video-recorded and rated blind to clinical status or diagnoses. Evaluation includes temporal-spatial errors as well as content errors. The total score of the 48 items may achieve 240. Further details are given in the supplementary material. Even though the TULIA contains six gesture categories, the neuroimaging analyses focused only on meaningful gestures (transitive and intransitive) performed on verbal command and imitation of meaningless gestures.

\subsection{Neuroimaging}

Structural imaging was performed with a 3D-T1-weighted (Modified Driven Equilibrium Fourier Transform Pulse Sequence; MDEFT) (Deichmann, Schwarzbauer, \& Turner, 2004) sequence on a 3-T scanner (Siemens Magnetom Trio; Siemens Medical Solutions, Erlangen, Germany), providing 176 sagittal slices with $256 \times 256$ matrix points with a field of view (FOV) of $256 \times 256$, yielding a nominal isotopic resolution of $1 \mathrm{~mm}^{3}$ (i.e. $1 \mathrm{~mm} \times 1 \mathrm{~mm} \times$ $1 \mathrm{~mm}$ ). Further scan parameters were $7.92 \mathrm{~ms}$ repetition time (TR), $2.48 \mathrm{~ms}$ echo time (TE) and a flip angle of $16^{\circ}$ (FA).

We processed structural images using SPM8 (Wellcome Trust Center for Neuroimaging, London; http://www.fil.ion.ucl.ac.uk/spm) using standard procedures of voxel based morphometry as implemented in SPM8 (Ashburner \& Friston, 2000). Images were normalized, segmented into tissue classes, modulated and smoothed with $8 \mathrm{~mm}$ full-width at half maximum (FWHM) kernel (Honea, Crow, Passingham, \& Mackay, 2005).

In detail, for segmentation the high-resolution cerebral MRI for each subject is classified into different tissue types such as the gray matter (GM), the white matter (WM) and the cerebral spinal fluid (CSF) according to prior-information on the distribution of tissues in normalized images. The segmentation procedure is based on a "modified gaussian mixture model" in which each voxel is assigned a probability value that represents the likelihood of belonging to one of the tissue class (Ashburner \& Friston, 2000, 2005).

Normalization transforms the native MR images into the Montreal Neurological Institute (MNI) template brain space using a 12-parameter affine transformation and a non-linear registration to account for gross differences in head shape (Ashburner \& Friston, 2000, 2005). Finally, the images are spatially smoothed with a Gaussian-kernel with a Full width at half Maximum (FWHM) of $8 \mathrm{~mm}$ in order to account for variability in spatial normalization and variability in registration of images of different subjects (Ashburner \& Friston, 2000, 2005; Honea et al., 2005). Normalized, modulated and smoothed images of gray matter were used in 
further analyses. Anatomical labelling within the MNI space was performed using automated anatomical labeling (aal) in SPM (Tzourio-Mazoyer et al., 2002)

\subsection{Statistical analyses}

\subsubsection{Analyses of clinical data and gesture tests}

Demographic and clinical parameters were compared between groups using t-Tests, $\mathrm{X}^{2}$-tests or ANOVAs where appropriate using the SPSS statistics package 22. TULIA scores were entered into a repeated measures ANOVA with factors domain (imitation, pantomime), category (intransitive, transitive, meaningless) and group (patients, controls); GreenhouseGeisser correction was applied.

\subsubsection{Analyses of structural imaging data}

The main analyses focused on the brain-behavior associations in SPM8 on the group level (schizophrenia patients with gesture deficits vs. schizophrenia patients without gesture deficits vs. healthy controls). Patients were classified according to the published cut-off scores for the TULIA test (Walther et al., 2013a). As patients' performance in the gesture tests may vary among the gesture domain or category, group allocation was performed twice: first for performance of meaningful gestures on command (TULIA score: pantomime transitive + pantomime intransitive) and second for imitation of meaningless gestures. We used two whole brain ANCOVAs for three groups (schizophrenia patients with gesture deficits vs. schizophrenia patients without gesture deficits vs. healthy controls) corrected for age, education and total intracranial volume (TIV). For each gesture type_(meaningful gestures on verbal command and imitation of meaningless gestures) we calculated a separate ANCOVA. Within the context of the ANCOVA for meaningful gestures on verbal command, we also tested the GM volume differences between all patients and controls. For the ANCOVAs we applied a statistical threshold of $p<0.05$ (family wise error (FWE) corrected). Images were produced using SPM8 and MRIcron (Rorden, Karnath, \& Bonilha, 2007).

As an exploratory analysis, we tested the association of impaired performance of gestures on command and GM reductions for each meaningful gesture category separately in the patients. Therefore, we computed two multiple regression analyses of GM volume with age, education and TIV as covariates, one for performance of transitive gestures on command (TULIA $\underline{\text { transitive pantomime score) and one for performance of intransitive gestures on command }}$ (TULIA intransitive pantomime score). Finally, we tested whether any of the two gesture 
categories had specific GM correlates, calculating the multiple regression analyses again adding the score of the other category as covariate.

\section{Results}

\subsection{Behavioral and clinical data}

Demographic and clinical data are given in table 1. Schizophrenia patients performed poorer than controls in each TULIA domain and each TULIA gesture category. A repeated measures ANOVA of TULIA scores indicated significant effect of domain $\left(F_{1,89}=22.0, p<0.001\right)$ with superior imitation performance, category $\left(\mathrm{F}_{1.68,89}=50.4, p<0.001\right)$, and group $\left(F_{1,89}=\right.$ $22.5, p<0.001)$, as well as interactions of domain $\times \operatorname{group}\left(F_{1,89}=9.0, p=0.003\right)$ and domain $\times$ category $\times$ group $\left(F_{1.70,89}=5.0, p=0.012\right)$. Patients were particularly impaired in performance of gestures on verbal command (see Supplementary material figures 2 and 3). According to the TULIA cut-off scores (Walther et al., 2013a), 17 (38\%) of the patients had relevant impairments performing meaningful gestures on verbal command and $6(13 \%)$ in imitation of meaningless gestures. When we classified groups according to the performance of meaningful gestures on command into controls and patients with and without deficits, patients with deficits were older, less educated and presented with more severe motor signs (see table 4, Supplementary material). However, patients with and without deficits did not differ in terms of PANSS scores or medication dose. As imitation deficits were so rare, we omitted the planned ANCOVA on imitation of meaningless gestures.

-- Please insert Table 1 here --

\subsection{Gray matter reductions in patients with defective gesture performance on} verbal command

The ANCOVA of gesture performance on verbal command over three groups identified significant clusters in the bilateral praxis network; the largest clusters within the left inferior frontal gyrus (IFG) pars orbitalis and in the right insula, but also in the right IPL and anterior cingulate cortex (ACC) (see table 2 and figure 1). Post-hoc contrasts demonstrated gray matter volume reductions in patients with gesture deficits compared to controls in 
predominantly left fronto-temporal regions particularly the left IFG pars orbitalis, right fronto-temporal brain regions including the right insula and temporal pole as well as the ACC. The second post-hoc contrast demonstrated reduced gray matter volume in patients with gesture deficits vs. patients without gesture deficits in the right insula, right IPL and right STG. The third post-hoc comparison of all patients vs. controls revealed a prominent loss of gray matter volume in left IFG and ACC in schizophrenia. Further volume differences were found in bilateral temporal lobes, right IFG, right insula and right precentral gyrus (see table 2 bottom).

-- Please insert Table 2 and Figure 1 here -

When the same ANCOVAs were run for the total TULIA imitation and pantomime scores, results remained similar with GM reductions in the left IFG, right insula and bilateral frontotemporal brain regions (see Supplementary material tables 7 and 8, figure 4).

\subsection{Associations of defective pantomime performance and brain structure in patients}

Within patients we computed exploratory multiple linear regression analyses of the association between gesture performance on verbal command and local gray matter volume for each of the two meaningful categories with age, education and TIV as covariates. Impaired performance of intransitive gestures on verbal command (TULIA intransitive pantomime) in patients was associated with reduced GM in the right insula and left hippocampus (see Table 3 top). Likewise, impaired performance of transitive gestures on verbal command (TULIA transitive pantomime) was linked to GM reduction in bilateral hippocampus, right IPL, SPL, and superior temporal gyrus (see table 3 bottom). When impairments of transitive gestures on command were covaried for the performance of intransitive gestures, age, education, and TIV, one cluster of reduced GM was detected in the

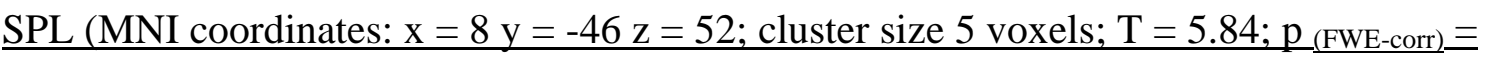
$\underline{0.020)}$.

-- Please insert Table 3- 


\section{Discussion}

This study investigated the neural correlates of impaired performance of hand and finger gestures in schizophrenia. In fact, schizophrenia served as a model of apraxia with discrete cerebral abnormalities and distinct gesture impairments. This approach adds to fMRI studies in healthy controls that lack behavioral impairments. Furthermore, the study extends results of lesion studies that are limited to macroscopic brain damage. We investigated schizophrenia patients with deficits in gesture performance on verbal command, patients without such deficits and healthy controls. The groups differed predominantly in GM within the ventrodorsal stream including left IFG confirming our hypotheses. Patients with gesture deficits differed substantially from controls in GM volume, but also from patients without deficits. As hypothesized, impaired performance of transitive (tool related) gestures on verbal command was linked to reduced GM in the dorso-dorsal stream, i.e. SPL. In contrast, we were unable to detect specific GM reductions in language related brain areas associated with impaired performance of intransitive (emblematic) gestures with conventional meaning on verbal command. In addition, the between defective gesture performance and brain structure could not be tested for imitation of meaningless gestures, because only six schizophrenia patients presented with clear deficits in this category.

\subsection{Comparison to brain lesion studies}

In line with the left-hemispheric lesion mapping studies in apraxia, schizophrenia patients with defective performance of meaningful gestures on command had reduced gray matter volumes in left IFG, right insula, bilateral SPL and multiple temporal areas compared to controls (Buxbaum et al., 2014; Goldenberg et al., 2007; Hoeren et al., 2014a; Manuel et al., 2013; Weiss et al., 2014). The most pronounced and focused alterations were detected in the left IFG pars orbitalis (see figure 1B). However, patients with gesture deficits had less GM volume than patients without deficits only within the right hemisphere of this praxis network. Therefore, the findings suggest that, although intact left IFG is critical for normal tool-related gesture performance on verbal command as previously demonstrated (Goldenberg et al., 2007), in schizophrenia volume loss in left IFG does not sufficiently account for overt impairments in performing meaningful gestures on verbal command.

The group differences in brain structure remain basically the same when considering the total TULIA pantomime score including meaningless gestures (see Supplementary Material table 7). Likewise, deficits in imitation performance were related to reduced GM in predominantly left IFG, insula and STG (see Supplementary Material table 8, figure 4), confirming findings 
from brain lesion studies (Goldenberg \& Karnath, 2006; Mengotti et al., 2013; Weiss et al., 2014). In summary, the location of gray matter reductions in schizophrenia patients presenting with behavioral gesture deficits matches the locations of brain damage in patients with apraxia, who present with more severe gesture deficits.

\subsection{Comparison to fMRI studies in healthy subjects}

As in comparison to lesion mapping studies, our findings in schizophrenia also parallel results of fMRI investigations of gesture performance in healthy subjects. This is particularly true for patients with deficits performing meaningful gestures on command, who have reduced GM in bilateral IFG, insula, SPL and multiple temporal as well as frontal brain regions (e.g. bilateral superior medial gyrus and anterior cingulate cortex: ACC) (Fridman et al., 2006; Kroliczak \& Frey, 2009; Vry et al., 2015). In addition, defective gesture performance in schizophrenia was associated with GM reduction in the right hemisphere including right insula, IPL and middle frontal gyrus. However, in fMRI studies in controls gesture performance was linked to left lateralized brain activity in the praxis network (Fridman et al., 2006; Kroliczak \& Frey, 2009; Vry et al., 2015). Patients lacked such clear left-hemispheric lateralization, even though the left IFG was the most affected brain region when contrasting patients with gesture deficits and controls. Moreover, patients with impaired gesture performance on verbal command had reduced GM volume only in small clusters of the IPL and SPL, areas which were frequently reported to be strongly engaged in gesture performance in healthy subjects (Bohlhalter et al., 2009; Horan et al., 2014; Johnson-Frey et al., 2005; Kroliczak \& Frey, 2009; Vry et al., 2015).

\subsection{Semantic categories}

The semantic context of gestures is probably reflected in the neural representations of gesture production. Indeed, lesion studies suggest a link between language and meaningful gestures via semantic processing (Goldenberg \& Randerath, 2015; Mengotti et al., 2013; Weiss et al., 2014). Instead, meaningless gestures are novel movements and therefore the imitation of meaningless gestures may not rely on stored semantic or action knowledge.

The primary aim of the current study was to test GM alterations in schizophrenia patients with particularly poor performance of meaningful gestures on verbal command, which include intransitive and transitive gestures of the TULIA. Due to a limited number of subjects presenting deficits, however, we missed to test the GM alterations associated with impaired 
imitation of meaningless gestures. Likewise, a comparison of GM alterations associated with either meaningless or meaningful gesture impairments in schizophrenia cannot be addressed in this report. Our secondary aim was to investigate the associations of altered GM volume and impaired performance of meaningful gestures on verbal command in patients for each of the two meaningful gesture categories.

Intransitive gestures have a conventionally agreed meaning without object relation and contribute substantially to nonverbal communication. Semantic knowledge is therefore critical to plan and execute intransitive gestures. We thus hypothesized that the language system was critical for performance of intransitive gestures. In fact, the use of metaphoric gestures, which are summarized as intransitive, has been associated with the volume of the STG in healthy subjects (Bernard, Millman, \& Mittal, 2015). Indeed, we found that schizophrenia patients with poor performance of meaningful gestures on command (transitive and intransitive) had reduced GM volumes in language related brain areas such as bilateral IFG and STG. Likewise, exploratory multiple regression analysis of intransitive gestures detected an association with GM volume of the right insula and the left hippocampus. But no cluster of the latter analysis survived when correcting the multiple regression analysis for the performance of transitive gestures.

Transitive gestures refer to the demonstration of object use (mainly tools) without the object. For this skill abstract motor planning and action knowledge are required. Performance of transitive gestures on command was associated with brain activity in bilateral IFG, PMd, SPL, IPL and STS with some inconsistencies across studies (Bohlhalter et al., 2009; Kroliczak \& Frey, 2009; Vry et al., 2015). Likewise, lesion studies demonstrated that IFG, insula and IPL were critical for transitive gestures on command (Buxbaum et al., 2014a; Hoeren et al., 2014b; Manuel et al., 2013; Weiss et al., 2014). In line with the above mentioned fMRI studies in healthy subjects and lesion studies, schizophrenia patients with deficits performing meaningful gestures on command had reduced GM in bilateral insula, IFG, STG, IPL and SPL. Moreover, multiple regression analysis indicated that in schizophrenia patients, performance of transitive gestures was linked to GM volume in right STG, IPL and SPL, but in addition also relied on bilateral hippocampus. The association with bilateral hippocampus could be explained by extra efforts in retrieving gesture representation from long-term memory. In fact, involvement of the hippocampus and anterior temporal lobe in transitive gestures on command has also been demonstrated in patients with severe aphasia, linking pantomime performance and linguistic abilities (Goldenberg \& Randerath, 2015). Finally, one cluster in the right SPL remained significant when performance of transitive gestures was 
covaried for the performance of intransitive gestures on command. SPL is considered to be part of the dorso-dorsal route (Vry et al., 2015). Thus, this SPL involvement suggests some specificity for transitive over intransitive gesture content.

\subsection{Relevance of findings for apraxia}

As noted above, we are aware of the distinct gesture tests applied in apraxia, which may use different terminology to describe the mode of elicitation and the semantic content of gestures (Bartolo, Cubelli, \& Della Sala, 2008; Goldenberg, 2009). Our main finding relates to meaningful gestures performed on verbal command. Within the dual route model of action, our results in schizophrenia are consistent with the notion that pantomime of tool-use is processed within the ventro-dorsal route, even though this was less evident for the posterior/parietal components (Hoeren et al., 2014b). In addition, patients with deficits performing meaningful gestures on command had reduced GM volume also in brain areas outside the ventro-dorsal route, namely in the temporal pole and dorsomedial prefrontal cortex, brain areas implicated in social and emotional processing (Amft et al., 2014; Olson, Plotzker, \& Ezzyat, 2007). Furthermore, the patients with gesture deficits had GM reductions within bilateral SPL, a key region of the dorso-dorsal route (Vry et al., 2015). In fact, the dorso-dorsal route was suggested to account for online motor control critical for imitation (Hoeren et al., 2014b).

Finally, the multiple regression analysis of gesture impairments and GM volume in both meaningful gesture categories in patients detected hippocampal contributions to the performance of gestures on verbal command. The hippocampus plays an important role in spatial memory processes (Eichenbaum \& Cohen, 2014). However, it is a matter of an ongoing debate whether memory-related brain areas are part of the two action routes. For instance, it has been hypothesized that the ventral route is a memory based system including the hippocampus-formation whereas the dorsal route does not have a long-term storage of information (Norman, 2002). In fact, in healthy controls planning of pantomime gestures relied on parahippocampal gyrus activity (Fridman et al., 2006). In summary, our data in schizophrenia patients challenge specific contributions of the two action routes to gesture performance. Instead, we found a predominance of fronto-temporal and limbic areas related to the performance of meaningful gestures on verbal command. 


\subsection{Comparison to fMRI studies in schizophrenia}

Studies on neural correlates of gesture performance in schizophrenia are missing. However, two recent fMRI investigations focused on imitation of meaningless finger movements. One study reported preserved neural activity during imitation, execution and observation of finger movements (Horan et al., 2014), while the other found reduced activation during imitation in the right IPL/STS in patients (Thakkar et al., 2014). In contrast, fMRI studies of gesture perception indicated preserved processing of concrete iconic gestures in schizophrenia, but lack of activation in abstract metaphoric gestures, which is due to a functional disconnection between superior temporal sulcus and bilateral IFG during processing of abstract gestures (Straube, Green, Sass, \& Kircher, 2014; Straube, Green, Sass, Kirner-Veselinovic, \& Kircher, 2013).

\subsection{Relevance of findlings for schizophrenia}

Findings of impaired hand gesture performance are consistent in two different samples (Walther et al., 2015; Walther et al., 2013a, 2013b). Furthermore, impaired imitation of hand and finger gestures has been established in schizophrenia (Matthews et al., 2013; Park et al., 2008). Gesture abnormalities have been reported in both subjects at risk for psychosis and schizophrenia patients (Lavelle et al., 2013; Millman et al., 2014; Mittal et al., 2006). In schizophrenia, poor gesture performance was closely linked to impaired nonverbal social perception and motor abnormalities (Walther et al., 2015). However, the neural correlates of this general impairment are yet to be explored. Our study demonstrates that the deficits in gesture performance in schizophrenia were linked to gray matter alterations in the same areas that are lesioned in apraxia. In fact, the types of gesture errors are similar between schizophrenia and apraxia, particularly in the TULIA pantomime domain (Walther et al., 2013b). The brain areas associated with poor gesture performance have been associated with schizophrenia in general (Haijma et al., 2013) or with impairments in social cognition (Benedetti et al., 2009; Regenbogen et al., 2015; Sui et al., 2015) and motor behavior (Bracht et al., 2013; Walther et al., 2011b). Moreover, insular dysfunction has been implicated in altered self-awareness and salience processing in schizophrenia (Uddin, 2015). Furthermore, the associations in predominantly frontal brain alterations (e.g. bilateral superior medial gyrus and ACC) are in line with our initial behavioral finding of a link between pantomime performance and poor frontal lobe function in schizophrenia (Walther et al., 2013b). The frontal lobe is relevant for higher order motor control including planning and execution (Miller \& Cohen, 2001). In particular, structural and functional alterations within the ACC have repeatedly been reported in schizophrenia (Haijma et al., 2013) and may contribute to 
aberrant motor behavior (Bracht et al., 2013; Walther et al., 2011a, 2011b and for review see Walther, 2015)). Thus, reduced gray matter in the ACC may lead to poor motor control, which impairs gesture performance in schizophrenia (Walther et al., 2015).

\subsection{Limitations}

We applied a validated test of gesture performance in a large sample of schizophrenia patients. Most of the subjects were medicated with antipsychotics. While antipsychotics might have an effect on gesture performance the dosage of current antipsychotic use was not related to gesture performance in schizophrenia (Walther et al., 2015). In our patients, distribution of gesture deficits was partially overlapping. Most of the subjects with imitation deficits (four out of six) also presented defective performance of gestures on verbal command (see Table 6, Supplementary material). Thus, it was impossible to completely separate effects of different modes of gesture elicitation (imitation vs. on verbal command). In addition, the study lacked sufficient statistical power to directly test the correlations of GM volume and each of the gesture categories (intransitive, transitive and meaningless). A general limitation of VBM GM analyses is the limited interpretation of putative underlying causes of reduced GM volume.

\subsection{Conclusions}

Impaired gesture performance in schizophrenia was associated with reduced gray matter volume in bilateral, predominantly left praxis network. Consistent with lesion studies gesture performance in schizophrenia relies on structural integrity of left IFG. In addition, right insula is mainly involved, which is thought to be important in self-awareness and salience detection often disturbed in schizophrenia. Performance of meaningful gestures on verbal command relied on but was not limited to the ventro-dorsal route, including language related brain areas. Therefore, impaired gesture performance in schizophrenia was associated with structural brain alterations in typical areas of brain lesions in apraxia. The deviation from findings in apraxia may result from an intermediate position between healthy and lesioned brains, or from additional specific processes in schizophrenia.

\section{Role of the fundling source}

This study was supported by grants from the Bangerter-Rhyner Foundation and the Swiss National Science Foundation (SNF, grant \# 152619/1). The founding sources had no role in the study at any phase. 


\section{Acknowledgement}

The authors would like to thank Dr. Corrado Bernasconi for statistical advice.

\section{References}

Amft, M., Bzdok, D., Laird, A. R., Fox, P. T., Schilbach, L., \& Eickhoff, S. B. (2014). Definition and characterization of an extended social-affective default network. Brain Struct Funct. doi: 10.1007/s00429-013-0698-0

Andreasen, N. C., Flaum, M., \& Arndt, S. (1992). The Comprehensive Assessment of Symptoms and History (CASH). An instrument for assessing diagnosis and psychopathology. Arch Gen Psychiatry, 49(8), 615-623.

Ashburner, J., \& Friston, K. J. (2000). Voxel-based morphometry--the methods. Neuroimage, 11(6 Pt 1), 805-821. doi: 10.1006/nimg.2000.0582 S10538119(00)90582-2 [pii]

Ashburner, J., \& Friston, K. J. (2005). Unified segmentation. Neuroimage, 26(3), 839-851. doi: 10.1016/j.neuroimage.2005.02.018

Bartolo, A., Cubelli, R., \& Della Sala, S. (2008). Cognitive approach to the assessment of limb apraxia. Clin Neuropsychol, 22(1), 27-45. doi: 10.1080/13854040601139310

Benedetti, F., Bernasconi, A., Bosia, M., Cavallaro, R., Dallaspezia, S., Falini, A., . . Smeraldi, E. (2009). Functional and structural brain correlates of theory of mind and empathy deficits in schizophrenia. Schizophr Res, 114(1-3), 154-160. doi: 10.1016/j.schres.2009.06.021

Binkofski, F., \& Buxbaum, L. J. (2013). Two action systems in the human brain. Brain Lang, 127(2), 222-229. doi: 10.1016/j.bandl.2012.07.007

Bohlhalter, S., Hattori, N., Wheaton, L., Fridman, E., Shamim, E. A., Garraux, G., \& Hallett, M. (2009). Gesture subtype-dependent left lateralization of praxis planning: an event-related fMRI study. [Research Support, N.I.H., Intramural]. Cereb Cortex, 19(6), 1256-1262. doi: 10.1093/cercor/bhn168

Bora, E., Fornito, A., Radua, J., Walterfang, M., Seal, M., Wood, S. J., . . Pantelis, C. (2011). Neuroanatomical abnormalities in schizophrenia: a multimodal voxelwise metaanalysis and meta-regression analysis. Schizophr Res, 127(1-3), 46-57. doi: S0920-9964(11)00011-9 [pii] 10.1016/j.schres.2010.12.020

Bracht, T., Schnell, S., Federspiel, A., Razavi, N., Horn, H., Strik, W., ... Walther, S. (2013). Altered cortico-basal ganglia motor pathways reflect reduced volitional motor activity in schizophrenia. Schizophr Res, 143(2-3), 269-276. doi: S09209964(12)00677-9 [pii] 10.1016/j.schres.2012.12.004

Buxbaum, L. J., \& Kalenine, S. (2010). Action knowledge, visuomotor activation, and embodiment in the two action systems. [Review]. Ann N Y Acad Sci, 1191, 201218. doi: 10.1111/j.1749-6632.2010.05447.x

Buxbaum, L. J., Shapiro, A. D., \& Coslett, H. B. (2014). Critical brain regions for toolrelated and imitative actions: a componential analysis. [Research Support, N.I.H., Extramural]. Brain, 137(Pt 7), 1971-1985. doi: 10.1093/brain/awu111

Cartmill, E. A., Beilock, S., \& Goldin-Meadow, S. (2012). A word in the hand: action, gesture and mental representation in humans and non-human primates. Philos Trans R Soc Lond B Biol Sci, 367(1585), 129-143. doi: 10.1098/rstb.2011.0162

Deichmann, R., Schwarzbauer, C., \& Turner, R. (2004). Optimisation of the 3D MDEFT sequence for anatomical brain imaging: technical implications at 1.5 and $3 \mathrm{~T}$. 
Neuroimage, 21(2), 757-767. doi: 10.1016/j.neuroimage.2003.09.062 S105381190300613X [pii]

Eichenbaum, H., \& Cohen, N. J. (2014). Can We Reconcile the Declarative Memory and Spatial Navigation Views on Hippocampal Function? Neuron, 83(4), 764-770. doi: 10.1016/j.neuron.2014.07.032

Fahn, S., Elton, R. L., \& Members, U. P. (1987). Unified Parkinson's disease rating scale. In S. Fahn, C. D. Marsden, M. Goldstein \& D. B. Calne (Eds.), Recent developments in Parkinson's disease (Vol. 2). Florham Park, NJ: Macmillan Healthcare Information.

Fridman, E. A., Immisch, I., Hanakawa, T., Bohlhalter, S., Waldvogel, D., Kansaku, K., ... Hallett, M. (2006). The role of the dorsal stream for gesture production. Neuroimage, 29(2), 417-428. doi: 10.1016/j.neuroimage.2005.07.026

Goldenberg, G. (2009). Apraxia and the parietal lobes. Neuropsychologia, 47(6), 14491459. doi: 10.1016/j.neuropsychologia.2008.07.014

Goldenberg, G., Hermsdorfer, J., Glindemann, R., Rorden, C., \& Karnath, H. O. (2007). Pantomime of tool use depends on integrity of left inferior frontal cortex. Cereb Cortex, 17(12), 2769-2776. doi: bhm004 [pii] 10.1093/cercor/bhm004

Goldenberg, G., \& Karnath, H. O. (2006). The neural basis of imitation is body part specific. J Neurosci, 26(23), 6282-6287. doi: 26/23/6282 [pii]10.1523/JNEUROSCI.0638-06.2006

Green, M. F., Bearden, C. E., Cannon, T. D., Fiske, A. P., Hellemann, G. S., Horan, W. P., .. . Nuechterlein, K. H. (2012). Social cognition in schizophrenia, Part 1: performance across phase of illness. Schizophr Bull, 38(4), 854-864. doi: $10.1093 / \mathrm{schbul} / \mathrm{sbq} 171$

Guy, W. (1976). ECDEU Assessment Manual for Psychopharmacology. Rockville, MD: US Department of Health, Education and Welfare.

Haijma, S. V., Van Haren, N., Cahn, W., Koolschijn, P. C., Hulshoff Pol, H. E., \& Kahn, R. S. (2013). Brain volumes in schizophrenia: a meta-analysis in over 18000 subjects. Schizophr Bull, 39(5), 1129-1138. doi: 10.1093/schbul/sbs118

Hoeren, M., Kummerer, D., Bormann, T., Beume, L., Ludwig, V. M., Vry, M. S., ... Weiller, C. (2014a). Neural bases of imitation and pantomime in acute stroke patients: distinct streams for praxis. Brain, 137, 2796-2810. doi: 10.1093/brain/awu203

Hoeren, M., Kummerer, D., Bormann, T., Beume, L., Ludwig, V. M., Vry, M. S., . . Weiller, C. (2014b). Neural bases of imitation and pantomime in acute stroke patients: distinct streams for praxis. [Research Support, Non-U.S. Gov't]. Brain, 137(Pt 10), 2796-2810. doi: 10.1093/brain/awu203

Honea, R., Crow, T. J., Passingham, D., \& Mackay, C. E. (2005). Regional deficits in brain volume in schizophrenia: a meta-analysis of voxel-based morphometry studies. Am J Psychiatry, 162(12), 2233-2245. doi: 162/12/2233 [pii] 10.1176/appi.ajp.162.12.2233

Horan, W. P., Iacoboni, M., Cross, K. A., Korb, A., Lee, J., Nori, P., ... Green, M. F. (2014). Self-reported empathy and neural activity during action imitation and observation in schizophrenia. [Research Support, N.I.H., Extramural

Research Support, Non-U.S. Gov't]. Neuroimage Clin, 5, 100-108. doi: 10.1016/j.nicl.2014.06.006

Johnson-Frey, S. H., Newman-Norlund, R., \& Grafton, S. T. (2005). A distributed left hemisphere network active during planning of everyday tool use skills. [Research Support, N.I.H., Extramural Research Support, Non-U.S. Gov't Research Support, U.S. Gov't, P.H.S.]. Cereb Cortex, 15(6), 681-695. doi: 10.1093/cercor/bhh169

Kay, S. R., Fiszbein, A., \& Opler, L. A. (1987). The positive and negative syndrome scale (PANSS) for schizophrenia. Schizophr Bull, 13(2), 261-276. 
Kroliczak, G., \& Frey, S. H. (2009). A common network in the left cerebral hemisphere represents planning of tool use pantomimes and familiar intransitive gestures at the hand-independent level. [Research Support, N.I.H., Extramural]. Cereb Cortex, 19(10), 2396-2410. doi: 10.1093/cercor/bhn261

Lavelle, M., Healey, P. G., \& McCabe, R. (2013). Is nonverbal communication disrupted in interactions involving patients with schizophrenia? Schizophr Bull, 39(5), 11501158. doi: $10.1093 /$ schbul/sbs091

Manuel, A. L., Radman, N., Mesot, D., Chouiter, L., Clarke, S., Annoni, J. M., \& Spierer, L. (2013). Inter- and intrahemispheric dissociations in ideomotor apraxia: a largescale lesion-symptom mapping study in subacute brain-damaged patients. [Research Support, Non-U.S. Gov't]. Cereb Cortex, 23(12), 2781-2789. doi: $10.1093 /$ cercor/bhs280

Matthews, N., Gold, B. J., Sekuler, R., \& Park, S. (2013). Gesture imitation in schizophrenia. Schizophr Bull, 39(1), 94-101. doi: 10.1093/schbul/sbr062

Mengotti, P., Corradi-Dell'Acqua, C., Negri, G. A., Ukmar, M., Pesavento, V., \& Rumiati, R. I. (2013). Selective imitation impairments differentially interact with language processing. [Research Support, Non-U.S. Gov't]. Brain, 136(Pt 8), 2602-2618. doi: 10.1093/brain/awt194

Menz, M. M., McNamara, A., Klemen, J., \& Binkofski, F. (2009). Dissociating networks of imitation. Hum Brain Mapp, 30(10), 3339-3350. doi: 10.1002/hbm.20756

Millman, Z. B., Goss, J., Schiffman, J., Mejias, J., Gupta, T., \& Mittal, V. A. (2014). Mismatch and lexical retrieval gestures are associated with visual information processing, verbal production, and symptomatology in youth at high risk for psychosis. Schizophr Res, 158(1-3), 64-68. doi: 10.1016/j.schres.2014.06.007

Mittal, V. A., Tessner, K. D., McMillan, A. L., Delawalla, Z., Trotman, H. D., \& Walker, E. F. (2006). Gesture behavior in unmedicated schizotypal adolescents. J Abnorm Psychol, 115(2), 351-358. doi: 10.1037/0021-843X.115.2.351

Muhlau, M., Hermsdorfer, J., Goldenberg, G., Wohlschlager, A. M., Castrop, F., Stahl, R., ... Boecker, H. (2005). Left inferior parietal dominance in gesture imitation: an fMRI study. [Clinical Trial

Research Support, Non-U.S. Gov't]. Neuropsychologia, 43(7), 1086-1098. doi: 10.1016/j.neuropsychologia.2004.10.004

Norman, J. (2002). Two visual systems and two theories of perception: An attempt to reconcile the constructivist and ecological approaches. Behavioral and Brain Sciences, 25(1), 73-+.

Oldfield, R. C. (1971). The assessment and analysis of handedness: the Edinburgh inventory. Neuropsychologia, 9(1), 97-113.

Olson, I. R., Plotzker, A., \& Ezzyat, Y. (2007). The Enigmatic temporal pole: a review of findings on social and emotional processing. Brain, 130(Pt 7), 1718-1731. doi: $10.1093 /$ brain/awm052

Park, S., Matthews, N., \& Gibson, C. (2008). Imitation, simulation, and schizophrenia. Schizophr Bull, 34(4), 698-707. doi: sbn048 [pii] 10.1093/schbul/sbn048

Pazzaglia, M., Pizzamiglio, L., Pes, E., \& Aglioti, S. M. (2008). The sound of actions in apraxia. Curr Biol, 18(22), 1766-1772. doi: 10.1016/j.cub.2008.09.061

Peters, B. D., Blaas, J., \& de Haan, L. (2010). Diffusion tensor imaging in the early phase of schizophrenia: What have we learned? J Psychiatr Res. doi: S00223956(10)00155-X [pii] 10.1016/j.jpsychires.2010.05.003

Pinkham, A. E. (2014). Social cognition in schizophrenia. J Clin Psychiatry, 75 Suppl 2, 1419. doi: 10.4088/JCP.13065su1.04 
Regenbogen, C., Kellermann, T., Seubert, J., Schneider, D. A., Gur, R. E., Derntl, B., ... Habel, U. (2015). Neural responses to dynamic multimodal stimuli and pathology-specific impairments of social cognition in schizophrenia and depression. [Research Support, Non-U.S. Gov't]. Br J Psychiatry, 206(3), 198-205. doi: 10.1192/bjp.bp.113.143040

Rizzolatti, G., \& Matelli, M. (2003). Two different streams form the dorsal visual system: anatomy and functions. Exp Brain Res, 153(2), 146-157. doi: 10.1007/s00221003-1588-0

Rorden, C., Karnath, H. O., \& Bonilha, L. (2007). Improving lesion-symptom mapping. J Cogn Neurosci, 19(7), 1081-1088. doi: 10.1162/jocn.2007.19.7.1081

Schmidt, S. J., Mueller, D. R., \& Roder, V. (2011). Social cognition as a mediator variable between neurocognition and functional outcome in schizophrenia: empirical review and new results by structural equation modeling. Schizophr Bull, 37 Suppl 2, S41-54. doi: 10.1093/schbul/sbr079

Sheehan, D. V., Lecrubier, Y., Sheehan, K. H., Amorim, P., Janavs, J., Weiller, E., . . . Dunbar, G. C. (1998). The Mini-International Neuropsychiatric Interview (M.I.N.I.): the development and validation of a structured diagnostic psychiatric interview for DSM-IV and ICD-10. J Clin Psychiatry, 59 Suppl 20, 22-33;quiz 34-57.

Steele, J., Ferrari, P. F., \& Fogassi, L. (2012). From action to language: comparative perspectives on primate tool use, gesture and the evolution of human language. Philos Trans R Soc Lond B Biol Sci, 367(1585), 4-9. doi: 10.1098/rstb.2011.0295

Straube, B., Green, A., Sass, K., \& Kircher, T. (2014). Superior temporal sulcus disconnectivity during processing of metaphoric gestures in schizophrenia. Schizophr Bull, 40(4), 936-944. doi: 10.1093/schbul/sbt110

Straube, B., Green, A., Sass, K., Kirner-Veselinovic, A., \& Kircher, T. (2013). Neural integration of speech and gesture in schizophrenia: evidence for differential processing of metaphoric gestures. Hum Brain Mapp, 34(7), 1696-1712. doi: 10.1002/hbm.22015

Sui, J., Pearlson, G. D., Du, Y., Yu, Q., Jones, T. R., Chen, J., . . Calhoun, V. D. (2015). In Search of Multimodal Neuroimaging Biomarkers of Cognitive Deficits in Schizophrenia. Biol Psychiatry. doi: 10.1016/j.biopsych.2015.02.017

Thakkar, K. N., Peterman, J. S., \& Park, S. (2014). Altered brain activation during action imitation and observation in schizophrenia: a translational approach to investigating social dysfunction in schizophrenia. [Research Support, N.I.H., Extramural Research Support, Non-U.S. Gov't]. Am J Psychiatry, 171(5), 539-548. doi: 10.1176/appi.ajp.2013.13040498

Tzourio-Mazoyer, N., Landeau, B., Papathanassiou, D., Crivello, F., Etard, O., Delcroix, N., . .. Joliot, M. (2002). Automated anatomical labeling of activations in SPM using a macroscopic anatomical parcellation of the MNI MRI single-subject brain. Neuroimage, 15(1), 273-289. doi: 10.1006/nimg.2001.0978

Uddin, L. Q. (2015). Salience processing and insular cortical function and dysfunction. Nat Rev Neurosci, 16(1), 55-61. doi: 10.1038/nrn3857

Vanbellingen, T., Kersten, B., Van Hemelrijk, B., Van de Winckel, A., Bertschi, M., Muri, R., . .. Bohlhalter, S. (2010). Comprehensive assessment of gesture production: a new test of upper limb apraxia (TULIA). [Evaluation Studies Research Support, NonU.S. Gov't]. Eur J Neurol, 17(1), 59-66. doi: 10.1111/j.1468-1331.2009.02741.x

Vanbellingen, T., Schumacher, R., Eggenberger, N., Hopfner, S., Cazzoli, D., Preisig, B. C., . . . Muri, R. M. (2015). Different visual exploration of tool-related gestures in left hemisphere brain damaged patients is associated with poor gestural imitation. Neuropsychologia, 71, 158-164. doi: 10.1016/j.neuropsychologia.2015.04.001 
Vry, M. S., Tritschler, L. C., Hamzei, F., Rijntjes, M., Kaller, C. P., Hoeren, M., .. W Weiller, C. (2015). The ventral fiber pathway for pantomime of object use. Neuroimage, 106, 252-263. doi: 10.1016/j.neuroimage.2014.11.002

Walther, S. (2015). Psychomotor symptoms of schizophrenia map on the cerebral motor circuit. Psychiatry Res, 233(3), 293-298. doi: 10.1016/j.pscychresns.2015.06.010

Walther, S., Federspiel, A., Horn, H., Razavi, N., Wiest, R., Dierks, T., ... Muller, T. J. (2011a). Alterations of white matter integrity related to motor activity in schizophrenia. Neurobiol Dis, 42(3), 276-283. doi: S0969-9961(11)00029-5 [pii] 10.1016/j.nbd.2011.01.017

Walther, S., Federspiel, A., Horn, H., Razavi, N., Wiest, R., Dierks, T., ... Muller, T. J. (2011b). Resting state cerebral blood flow and objective motor activity reveal basal ganglia dysfunction in schizophrenia. Psychiatry Res, 192(2), 117-124. doi: 10.1016/j.pscychresns.2010.12.002

Walther, S., \& Mittal, V. A. (2016). Why We Should Take a Closer Look at Gestures. Schizophr Bull, 42(2), 259-261. doi: 10.1093/schbul/sbv229

Walther, S., Stegmayer, K., Sulzbacher, J., Vanbellingen, T., Muri, R., Strik, W., \& Bohlhalter, S. (2015). Nonverbal social communication and gesture control in schizophrenia. Schizophr Bull, 41(2), 338-345. doi: 10.1093/schbul/sbu222

Walther, S., Vanbellingen, T., Muri, R., Strik, W., \& Bohlhalter, S. (2013a). Impaired gesture performance in schizophrenia: Particular vulnerability of meaningless pantomimes. Neuropsychologia, 51(13), 2674-2678. doi: 10.1016/j.neuropsychologia.2013.08.017

Walther, S., Vanbellingen, T., Muri, R., Strik, W., \& Bohlhalter, S. (2013b). Impaired pantomime in schizophrenia: association with frontal lobe function. Cortex, 49(2), 520-527. doi: 10.1016/j.cortex.2011.12.008

Weiss, P. H., Ubben, S. D., Kaesberg, S., Kalbe, E., Kessler, J., Liebig, T., \& Fink, G. R. (2014). Where language meets meaningful action: a combined behavior and lesion analysis of aphasia and apraxia. Brain Struct Funct. doi: 10.1007/s00429-0140925-3 
Table 1. Demographic and clinical data.

\begin{tabular}{|c|c|c|c|c|c|c|}
\hline \multirow{3}{*}{ Gender (n) } & \multicolumn{2}{|c|}{ Controls } & \multicolumn{2}{|c|}{ Patients } & \multicolumn{2}{|c|}{ Tests } \\
\hline & \multicolumn{2}{|c|}{ Men/Women } & \multicolumn{2}{|c|}{ Men/Women } & & \\
\hline & \multicolumn{2}{|r|}{$26 / 18$} & \multicolumn{2}{|r|}{$28 / 17$} & & \\
\hline & $\mathrm{M}$ & SD & $\mathrm{M}$ & SD & & \\
\hline Age (y) & 38.77 & 13.58 & 38.24 & 11.37 & & \\
\hline Education (y) & 14.14 & 2.66 & 13.48 & 3.13 & & \\
\hline TULIA scores & & & & & $t$ & $p$ \\
\hline TULIA pantomime total & 112.05 & 4.95 & 98.79 & 17.70 & 4.83 & $<0.001$ \\
\hline pantomime intransitive & 38.82 & 1.45 & 35.73 & 6.21 & 3.24 & 0.002 \\
\hline pantomime transitive & 35.98 & 2.87 & 31.59 & 7.90 & 3.50 & 0.001 \\
\hline pantomime meaningless & 37.25 & 2.66 & 31.39 & 6.33 & 5.71 & $<0.001$ \\
\hline TULIA imitation total & 113.62 & 4.24 & 105.92 & 12.15 & 4.01 & $<0.001$ \\
\hline imitation intransitive & 39.24 & 1.32 & 36.18 & 4.07 & 4.79 & $<0.001$ \\
\hline imitation transitive & 35.57 & 3.36 & 33.10 & 5.83 & 2.45 & 0.017 \\
\hline imitation meaningless & 38.82 & 1.57 & 36.64 & 4.12 & 3.30 & 0.002 \\
\hline
\end{tabular}

TULIA - Test of Upper Limb Apraxia 
Table 2. ANCOVA of GM and pantomime performance: comparisons of controls vs. patients with vs. patients without pantomime deficits

\begin{tabular}{|c|c|c|c|c|c|c|}
\hline \multirow[t]{2}{*}{ Contrast/Brain region } & \multirow[b]{2}{*}{$\mathbf{K}$} & \multirow[b]{2}{*}{$\mathbf{F} / \mathbf{t}$} & \multirow[b]{2}{*}{$p_{\text {(FWE corr) }}$} & \multicolumn{3}{|c|}{ MNI coordinates } \\
\hline & & & & $\mathbf{x}$ & $\mathbf{y}$ & $\mathbf{z}$ \\
\hline \multicolumn{7}{|l|}{ F-Test Pantomime } \\
\hline \multirow{2}{*}{$\begin{array}{l}\text { Left IFG pars orbitalis extending } \\
\text { to left temporal pole }\end{array}$} & 159 & $F=19.05$ & 0.003 & -42 & 22 & -8 \\
\hline & & $F=16.59$ & 0.015 & -42 & 16 & -16 \\
\hline Left temporal pole & 19 & $F=16.50$ & 0.016 & -32 & 22 & -32 \\
\hline Left superior parietal lobe & 1 & $F=14.86$ & 0.044 & -4 & -66 & 58 \\
\hline \multirow[t]{3}{*}{ Right insula } & 348 & $F=24.62$ & $<0.001$ & 40 & 0 & 8 \\
\hline & & $F=21.90$ & 0.001 & 42 & 20 & 0 \\
\hline & & $F=19.74$ & 0.002 & 48 & -6 & 4 \\
\hline Right middle temporal gyrus & 53 & $F=19.37$ & 0.003 & 62 & -6 & -16 \\
\hline Right inferior parietal lobe (IPL) & 14 & $F=17.62$ & 0.008 & 64 & -46 & 28 \\
\hline Right superior temporal gyrus & 28 & $F=17.62$ & 0.009 & 48 & -26 & 12 \\
\hline \multirow{3}{*}{$\begin{array}{l}\text { Right/Left superior medial } \\
\text { gyrus extending to anterior } \\
\text { cingulate cortex (ACC) }\end{array}$} & 136 & $F=16.55$ & 0.015 & 4 & 56 & -2 \\
\hline & & $F=16.18$ & 0.019 & 4 & 52 & 6 \\
\hline & & $F=16.04$ & 0.021 & -4 & 52 & 12 \\
\hline Right parahippocampal gyrus & 26 & $F=16.36$ & 0.017 & 24 & 12 & -26 \\
\hline Right insula & 11 & $\mathrm{~F}=16.19$ & 0.019 & 42 & -14 & -4 \\
\hline Right superior frontal gyrus & 3 & $\mathrm{~F}=15.73$ & 0.025 & 28 & 58 & 8 \\
\hline Right inferior temporal gyrus & 2 & $\mathrm{~F}=15.50$ & 0.029 & 64 & -18 & -28 \\
\hline Right middle orbital gyrus & 1 & $\mathrm{~F}=14.75$ & 0.047 & 2 & 46 & 0 \\
\hline \multicolumn{7}{|l|}{ patients with deficits $<$ controls } \\
\hline \multirow{3}{*}{$\begin{array}{l}\text { Left IFG pars orbitalis extending } \\
\text { to left temporal pole }\end{array}$} & 916 & 6.15 & $<0.001$ & -42 & 22 & -8 \\
\hline & & 5.75 & 0.001 & -42 & 16 & -16 \\
\hline & & 5.71 & 0.002 & -32 & 22 & -32 \\
\hline Left middle occipital gyrus & 9 & 5.17 & 0.011 & -30 & -84 & 38 \\
\hline Left superior medial gyrus & 11 & 5.16 & 0.012 & -8 & 56 & 40 \\
\hline Left superior parietal lobe (SPL) & 20 & 5.08 & 0.015 & -2 & -68 & 56 \\
\hline Left middle temporal gyrus & 27 & 5.02 & 0.019 & -70 & -34 & -4 \\
\hline Left middle temporal gyrus & 4 & 4.76 & 0.045 & -50 & -26 & -4 \\
\hline
\end{tabular}




\begin{tabular}{|c|c|c|c|c|c|c|}
\hline \multirow{3}{*}{$\begin{array}{l}\text { Right insula extending to } \\
\text { superior temporal gyrus }\end{array}$} & 976 & 6.68 & $<0.001$ & 40 & 0 & 8 \\
\hline & & 6.44 & $<0.001$ & 42 & 20 & 0 \\
\hline & & 6.13 & $<0.001$ & 46 & -6 & 4 \\
\hline \multirow[t]{3}{*}{ Right middle temporal gyrus } & 177 & 6.15 & $<0.001$ & 62 & -4 & -16 \\
\hline & & 5.33 & 0.006 & 58 & 4 & -22 \\
\hline & & 5.14 & 0.013 & 58 & -14 & -8 \\
\hline \multirow{3}{*}{$\begin{array}{l}\text { Right/Left superior medial } \\
\text { gyrus extending to anterior } \\
\text { cingulate cortex (ACC) }\end{array}$} & 834 & 5.75 & 0.001 & 4 & 56 & -2 \\
\hline & & 5.69 & 0.002 & 4 & 52 & 6 \\
\hline & & 5.66 & 0.002 & -4 & 52 & 12 \\
\hline Right temporal pole & 135 & 5.58 & 0.003 & 24 & 10 & -28 \\
\hline Right superior frontal gyrus & 39 & 5.45 & 0.004 & 28 & 58 & 8 \\
\hline Right precentral gyrus (PMd) & 23 & 5.35 & 0.006 & 48 & -16 & 58 \\
\hline Right inferior temporal gyrus & 19 & 5.28 & 0.008 & 64 & -18 & -28 \\
\hline $\begin{array}{l}\text { Right superior parietal gyrus } \\
\text { (SPL) }\end{array}$ & 17 & 5.20 & 0.010 & 14 & -34 & 50 \\
\hline \multirow[t]{2}{*}{ Right cerebellum } & 27 & 5.15 & 0.012 & 20 & -94 & -24 \\
\hline & & 4.97 & 0.023 & 12 & -96 & -20 \\
\hline $\begin{array}{l}\text { Right inferior frontal gyrus pars } \\
\text { opercularis }\end{array}$ & 14 & 5.08 & 0.015 & 50 & 10 & 32 \\
\hline $\begin{array}{l}\text { Right Inferior frontal gyrus pars } \\
\text { opercularis }\end{array}$ & 33 & 5.05 & 0.017 & 56 & 12 & 10 \\
\hline Right middle frontal gyrus & 17 & 5.04 & 0.018 & 44 & 46 & 16 \\
\hline Right cerebellum & 29 & 5.01 & 0.020 & 36 & -38 & -30 \\
\hline Right middle frontal gyrus & 4 & 4.85 & 0.034 & 38 & 50 & 24 \\
\hline Right middle orbital gyrus & 3 & 4.84 & 0.035 & 42 & 56 & 2 \\
\hline $\begin{array}{l}\text { Right middle cingulate cortex, } \\
\text { posterior rostral cingulate } \\
\text { motor area (RCZp) }\end{array}$ & 1 & 4.79 & 0.041 & 8 & 24 & 44 \\
\hline Right middle frontal gyrus & 1 & 4.77 & 0.043 & 38 & 32 & 40 \\
\hline Right superior medial gyrus & 1 & 4.76 & 0.045 & 8 & 58 & 36 \\
\hline Right inferior parietal lobe (IPL) & 2 & 4.75 & 0.046 & 64 & -46 & 30 \\
\hline Right superior parietal lobe & 1 & 4.75 & 0.047 & 2 & -82 & 44 \\
\hline
\end{tabular}




\begin{tabular}{|c|c|c|c|c|c|c|}
\hline (SPL) & & & & & & \\
\hline Right middle frontal gyrus & 1 & 4.75 & 0.047 & 36 & -4 & 62 \\
\hline \multicolumn{7}{|c|}{ patients with deficits $<$ patients without deficits } \\
\hline \multirow[t]{2}{*}{ Right insula } & 94 & 5.65 & 0.001 & 38 & 0 & 8 \\
\hline & & 5.05 & 0.017 & 48 & -8 & 6 \\
\hline $\begin{array}{l}\text { Right inferior parietal lobe (IPL) } \\
\text { extending to superior temporal } \\
\text { gyrus }\end{array}$ & 31 & 5.63 & 0.002 & 64 & -46 & 28 \\
\hline Right superior temporal gyrus & 22 & 5.15 & 0.012 & 48 & -26 & 12 \\
\hline Right insula & 19 & 4.96 & 0.023 & 40 & 16 & 2 \\
\hline Right temporal pole & 3 & 4.86 & 0.032 & 56 & 6 & -30 \\
\hline $\begin{array}{l}\text { Right superior parietal lobe } \\
\text { (SPL) }\end{array}$ & 1 & 4.86 & 0.032 & 14 & -46 & 72 \\
\hline $\begin{array}{l}\text { Right superior parietal lobe } \\
\text { (SPL) }\end{array}$ & 2 & 4.82 & 0.034 & 6 & -80 & 50 \\
\hline Right superior temporal gyrus & 3 & 4.79 & 0.041 & 60 & -26 & 16 \\
\hline \multicolumn{7}{|l|}{ all patients $<$ controls } \\
\hline \multirow{3}{*}{$\begin{array}{l}\text { Left inferior frontal gyrus pars } \\
\text { orbitalis extending to temporal } \\
\text { pole }\end{array}$} & 264 & 5.78 & 0.001 & -44 & 22 & -8 \\
\hline & & 5.35 & 0.006 & -40 & 16 & -16 \\
\hline & & 4.86 & 0.032 & -52 & 18 & -10 \\
\hline \multirow{3}{*}{$\begin{array}{l}\text { Left/Right superior medial gyrus } \\
\text { extending to anterior cingulate } \\
\text { cortex (ACC) }\end{array}$} & 386 & 5.39 & 0.005 & -2 & 62 & 2 \\
\hline & & 5.27 & 0.008 & -2 & 52 & 8 \\
\hline & & 5.24 & 0.009 & -2 & 50 & 20 \\
\hline Left middle temporal gyrus & 6 & 4.97 & 0.022 & -48 & -52 & 4 \\
\hline Left middle temporal gyrus & 9 & 4.96 & 0.023 & -50 & -24 & -6 \\
\hline Left temporal pole & 24 & 4.93 & 0.026 & -34 & 22 & -30 \\
\hline Left middle occipital gyrus & 2 & 4.88 & 0.031 & -32 & -86 & 34 \\
\hline Left middle occipital gyrus & 1 & 4.78 & 0.042 & -30 & -84 & 38 \\
\hline Right middle temporal gyrus & 25 & 5.20 & 0.010 & 62 & -2 & -16 \\
\hline Right insula & 25 & 5.15 & 0.012 & 42 & 20 & 0 \\
\hline Right insula & 12 & 5.10 & 0.014 & 40 & 0 & 8 \\
\hline $\begin{array}{l}\text { Right inferior frontal gyrus pars } \\
\text { opercularis BA44 }\end{array}$ & 11 & 5.03 & 0.018 & 52 & 10 & 32 \\
\hline Right precentral gyrus (PMd) & 17 & 5.02 & 0.019 & 56 & -20 & 54 \\
\hline
\end{tabular}




\begin{tabular}{|l|r|r|r|r|r|r|}
\hline & & 4.95 & 0.024 & 46 & -16 & 60 \\
\hline Right insula & 20 & 5.00 & 0.020 & 46 & -4 & 2 \\
\hline Right superior parietal lobe (SPL) & 4 & 4.92 & 0.026 & 16 & -34 & 50 \\
\hline $\begin{array}{l}\text { Right inferior frontal gyrus pars } \\
\text { opercularis BA44 }\end{array}$ & 4 & 4.84 & 0.034 & 54 & 8 & 18 \\
\hline Right temporal pole & 2 & 4.78 & 0.042 & 58 & 6 & -20 \\
\hline
\end{tabular}

Covariates: age, education and TIV. $\mathrm{k}$ indicates cluster size. 
Table 3. Impaired gesturing on verbal command and GM reduction in patients.

\begin{tabular}{|c|c|c|c|c|c|c|c|}
\hline \multirow[t]{2}{*}{ Brain region } & \multicolumn{2}{|c|}{ Cluster level } & \multirow{2}{*}{$\begin{array}{l}\text { Peak level } \\
p \text { (FWE corr) }\end{array}$} & \multirow{2}{*}{\begin{tabular}{|l}
$\begin{array}{l}\text { Cluster } \\
\text { level }\end{array}$ \\
$p_{\text {(FWE corr) }}$
\end{tabular}} & \multicolumn{3}{|c|}{ MNI coordinates } \\
\hline & $\mathrm{k}$ & $\mathrm{T}$ & & & $\mathrm{x}$ & $\mathrm{y}$ & $\mathrm{z}$ \\
\hline \multicolumn{8}{|c|}{ intransitive gestures (conventional meaning) } \\
\hline Left hippocampus & 332 & 4.53 & 0.428 & 0.013 & -26 & -16 & -16 \\
\hline Right insula & 524 & 5.40 & 0.056 & 0.001 & 38 & 14 & -2 \\
\hline \multicolumn{8}{|c|}{ transitive gestures (tool related) } \\
\hline Left hippocampus & 992 & 5.00 & 0.162 & $<0.001$ & -26 & -16 & -16 \\
\hline Right hippocampus & 1074 & 5.00 & 0.160 & $<0.001$ & 14 & -6 & -18 \\
\hline Right superior temporal gyrus & 240 & 4.60 & 0.386 & 0.040 & 64 & -24 & 16 \\
\hline Right SPL & 2556 & 7.53 & $<0.001$ & $<0.001$ & 10 & -46 & 52 \\
\hline Right IPL & 576 & 5.10 & 0.124 & 0.001 & 20 & -74 & 48 \\
\hline
\end{tabular}

Covariates age, education and TIV. 
Figure legend

Figure 1 Group differences in GM volume according to gesture performance on verbal command (ANCOVA with covariates age, education, and TIV)

A: F-Test of group differences in the ANCOVA for three groups (controls, patients with deficits and patients without deficits in gesture performance on verbal command), rendered and sliced view. Color scale represents F-values.

B: T-test contrast patients with deficits vs. controls (rendered on the surface and sliced); left is left, numbers indicate the z position of the axial slices in MNI space, statistical threshold is $P_{\text {(FWE) }}<0.05$. Color scale represents T-values.

C: T-test contrast patients with deficits vs. patients without deficits (rendered on the surface and sliced); left is left, numbers indicate the $\mathrm{z}$ position of the axial slices in MNI space, statistical threshold is $P$ (FWE) $<0.05$. Color scale represents T-values. 


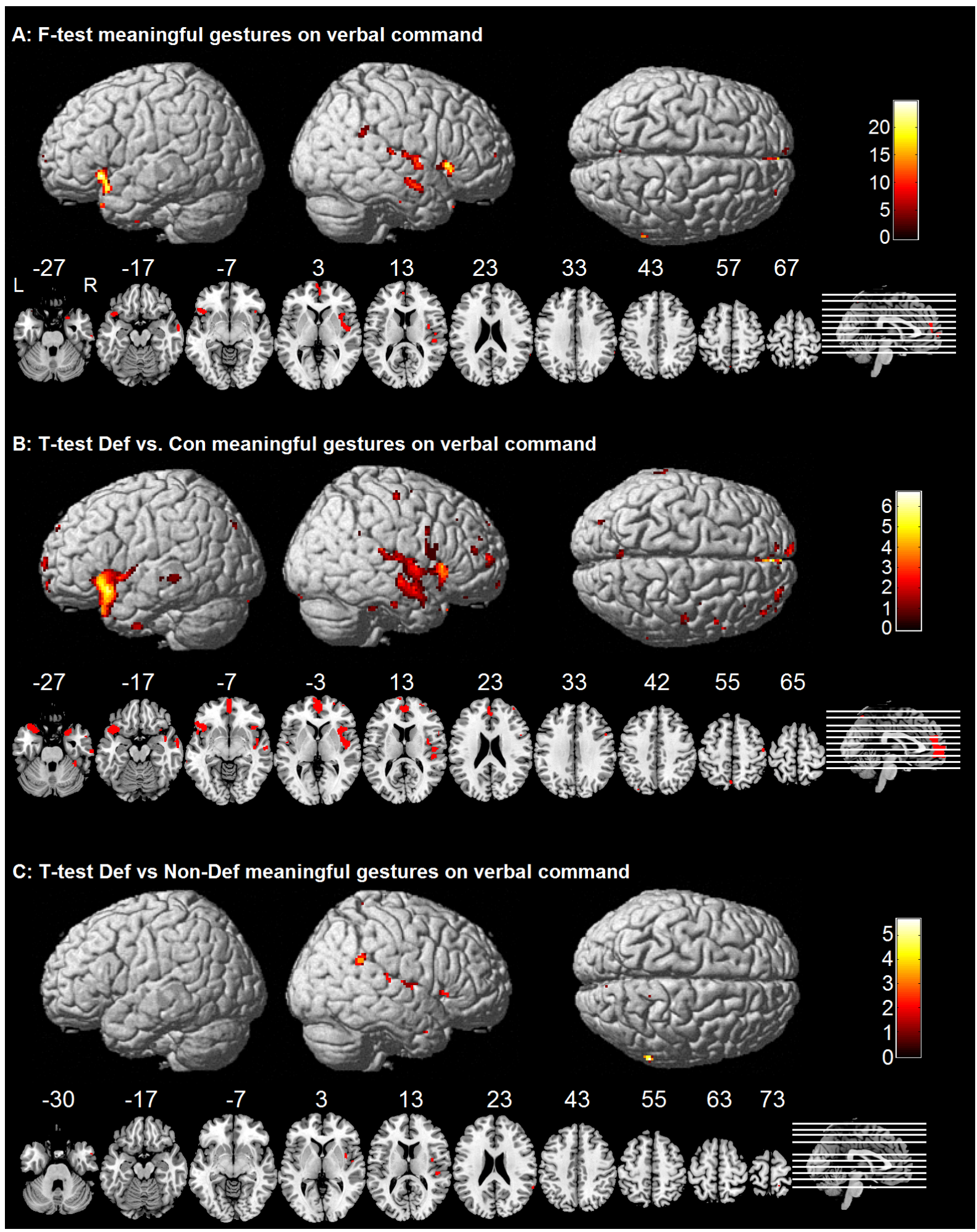

